\title{
SPECTROSCOPIC STUDY OF NGC 6119 - NGC 6120 SYSTEM
}

\author{
C. SURACE \\ Observatoire de Marseille \\ 2, place Le Verrier, 13248 Marseille cedex 4 (France) \\ AND \\ J. HECQUET AND M. AURIERE \\ Observatoire Midi Pyrennées \\ 14, Avenue E. Belin 31400 Toulouse (France)
}

NGC6119 and NGC6120 have been listed in Kiso catalog (Takase et al., 1987) as Sc and Irr galaxies. They have been selected by their clumpy features in order to investigate their nature and star formation history using slit spectroscopy. They have similar systemic velocities (respectively 9215 and $9200 \mathrm{~km} \cdot \mathrm{s}^{-1}$ ) and seem to be members of a low gravitational interacting system of 4 galaxies (with MCG-6-36-30 and NGC6122).

We have not got any photometry of NGC6119. However three emitting line regions have been spectroscopicaly resolved and present same characteristics as typical high ionized HII regions with solar metallicity.

NGC6120 have been classified as spiral galaxy. The three emission line regions spectra are characterized by low excitation Hydrogen Balmer emission lines superimposed to an old stellar component. Red color, old stellar component of central clump are consistent with Maehara observations (Maehara et al., 1988), and his definition of this clump as center of NGC6120. The disturbed spiral appearance of NGC6120 and the analysis of the spectrum at $33 \AA \mathrm{mm}^{-1}$ seem to confirm the hypothesis of a gas rich companion falling into the disk. The ages of the clumps were derived from Mas Hesse and Kunth models (1991) indicating a star formation older in this companion than in the other clumps.

\section{References}

Maehara H., Hamabe H., Bottinelli L., Gouguenheim L.,Takase B., 1988, PASJ, 40, 47

Mass Hess J.M., Kunth D., 1991, A\&AS, 88, 399

Takase B., Miyauchi-Isobe N., 1987, ATAO 2nd series, 21, 251 